\title{
KNOTS AND GRAVITY
}

\author{
TZE-CHUEN TOH ${ }^{1}$ and MALCOLM R. ANDERSON ${ }^{2}$
}

(Received 27 February 1995)

\begin{abstract}
In the loop representation theory of non-perturbative quantum gravity, gravitational states are described by functionals on the loop space of a 3-manifold. In the order to gain a deeper insight into the physical interpretation of loop states, a natural question arises: to wit, how are gravitations related to loops? Some light will be shed on this question by establishing a definite relationship between loops and 3-geometries of the 3-manifold.
\end{abstract}

\section{Introduction}

In the mid 80's, Ashtekar [1] formulated an alternative Hamiltonian approach to General Relativity. This led Rovelli et al. $[4,6]$ to formulate Quantum Gravity in terms of loops in a 3-manifold $\Sigma$. A loop in $\Sigma$ is just a closed curve starting and ending at the same point. An $n$-loop is the set $\left\{\gamma^{1}, \ldots, \gamma^{n}\right\}$ of $n$ loops $\gamma^{i}$ in $\Sigma$.

Very briefly, the loop representation of Quantum Gravity describes gravitational states via complex functionals $\Psi$ on the space of multi-loops of $\Sigma$. The functionals describing the physical states of gravity satisfy

(1) $\Psi$ is a constant on knot classes;

(2) $\Psi$ has support on smooth multi-loops without intersections. ${ }^{3}$

The physical interpretation still remains an open question. However, Rovelli [5, p. 1661] sketched a heuristic argument revealing the emergence of a discrete structure to space-time at the Plank scale. It will be tersely shown here that certain choices of $\aleph_{0}$-loops relate to 3 -geometries in a natural way. This in turn yield a deeper insight into the way loops and gravity are related.

\footnotetext{
'Department of Theoretical Physics, Research School of Physical Science and Engineering, The Australian National University, Canberra, Australian Capital Territory 0200, Australia.

${ }^{2}$ School of Engineering and Mathematics, Edith Cowan University, Joondalup Drive, Joondalup, Western Australia 6027, Australia.

(C) Australian Mathematical Society 1999, Serial-fee code 0334-2700/99

${ }^{3}$ Extensions to piecewise smooth loops have also been done. Refer to $[2,3]$ for more details.
} 


\section{Definitions and notations}

All loops considered here will be piecewise smooth in some fixed 3-manifold $\Sigma$, where $\Sigma$ is assumed to be smooth, closed, compact, orientable and Riemannian. By a Riemannian 3-metric $q$ on $\Sigma$ is meant a symmetric, covariant 2-tensor that is positivedefinite at each point $x \in \Sigma$. The space of Riemannian 3-metrics on $\Sigma$ will be denoted by $\Gamma_{2}^{+}$and the space of (Riemannian) 3-geometries of $\Sigma$ by $\mathscr{Q} \stackrel{\text { def }}{=} \Gamma_{2}^{+} / \operatorname{Diff}^{+}(\Sigma)$, where $\operatorname{Diff}^{+}(\Sigma)$ denotes the group of smooth, orientation-preserving diffeomorphisms on $\Sigma$ and a 3-geometry is defined by the equivalence class $[q] \stackrel{\text { def }}{=}\left\{f^{*} q \mid f \in \operatorname{Diff}^{+}(\Sigma)\right\}$ of metrics $q \in \Gamma_{2}^{+}$related by coordinate transformations. The space $\Gamma_{2}^{+}$is endowed with the compact $C^{\infty}$-topology and $\mathscr{Q}$ is given the quotient topology.

Now, given curves $\gamma, \eta: I \rightarrow \Sigma, I \stackrel{\text { def }}{=}[0,1]$, with $\gamma(0)=\gamma(1)$, define $\gamma * \eta$ by

$$
\gamma * \eta(t)= \begin{cases}\gamma(2 t) & \text { for } 0 \leqq t \leqq \frac{1}{2} \\ \eta(2 t-1) & \text { for } \frac{1}{2} \leqq t \leqq 1\end{cases}
$$

Call a curve $\gamma$ a $q$-geodesic if $\gamma$ is a (parametrized) geodesic in $\Sigma$ with respect to $q \in \Gamma_{2}^{+}$.

DEFINITION 2.1. $\gamma$ is a piecewise geodesic loop if $\exists q \in \Gamma_{2}^{+}$and $n$ smooth $q$ geodesics $\gamma_{1}, \ldots, \gamma_{n}: I \rightarrow \Sigma$ such that $\gamma=\gamma_{1} * \cdots * \gamma_{n}$.

Let $D_{\Sigma} \subset \Sigma$ denote a countably dense subset and let $\mathscr{M}_{\infty}\left[\Gamma_{2}^{+}\right]$, for each $q \in \Gamma_{2}^{+}$, be the set of $\aleph_{0}$-loops $\gamma=\left\{\gamma^{i}: i \in \mathbb{N}\right\}$ such that

(1) $\forall i, \gamma^{i}$ is a piecewise, affinely parametrized, $q$-geodesic loop in $\Sigma$;

(2) $\gamma$ is in bijective $e^{4}$ correspondence with $D_{\Sigma}$ under $\gamma^{i} \mapsto \gamma^{i}(0)$.

It is easy to see that conditions (1) and (2) together imply that each $\gamma$ corresponds to a unique 3-geometry $\left[q_{\gamma}\right] \in \mathscr{Q}$. For suppose $\gamma$ is both a $q$-geodesic loop as well as a $q^{\prime}$-geodesic loop. Then, with respect to charts $U_{\alpha}$,

$$
\left(\ddot{\gamma}_{\alpha}^{i}\right)^{l}+\Gamma_{\alpha}(q)_{k j}^{l}\left(\dot{\gamma}_{\alpha}^{i}\right)^{k}\left(\dot{\gamma}_{\alpha}^{i}\right)^{j} \stackrel{\text { a.e. }}{=} 0 \text { and }\left(\ddot{\gamma}_{\alpha}^{i}\right)^{l}+\Gamma_{\alpha}\left(q^{\prime}\right)_{k j}^{l}\left(\dot{\gamma}_{\alpha}^{i}\right)^{k}\left(\dot{\gamma}_{\alpha}^{i}\right)^{j} \stackrel{\text { a.e. }}{=} 0
$$

on $\gamma^{i}(I) \cap U_{\alpha}$ for each $i$ (no summation over $\alpha$ ), where a.e. means that the equality holds on $I-\left\{t_{1}, \ldots, t_{n}\right\}$, with $0 \leqq n<\infty$ and $n=0$ denoting the empty set. Hence, $\left(\Gamma_{\alpha}(q)_{k j}^{l}-\Gamma_{\alpha}\left(q^{\prime}\right)_{k j}^{l}\right)\left(\dot{\gamma}_{\alpha}^{i}\right)^{k}\left(\dot{\gamma}_{\alpha}^{i}\right)^{j} \stackrel{\text { a.e. }}{=} 0 \forall \gamma^{i} \in \gamma$ and $\alpha$. Thus by (2), $\Gamma(q)_{k j}^{l}(x) \equiv$ $\Gamma\left(q^{\prime}\right)_{k j}^{l}(x)$ on a dense subset of $\Sigma$ as $\overline{U\left\{\gamma^{i}(I) \mid \gamma^{i} \in \gamma\right\}} \equiv \Sigma$ by (2). So, invoking the continuity of $\Gamma(h)$ for $h=q, q^{\prime}$, it follows at once that $\Gamma(q) \equiv \Gamma\left(q^{\prime}\right)$ on $\Sigma$. Now, with respect to local coordinate basis, $\Gamma(q)_{k j}^{l}=\frac{1}{2} q^{i h}\left(\partial_{k} q_{h j}+\partial_{j} q_{h k}-\partial_{h} q_{k j}\right)$ (and likewise

${ }^{4}$ This condition may be relaxed to a surjection. 
for $q^{\prime}$ ); consequently, $q$ and $q^{\prime}$ are related homothetically; that is, $\exists c>0$ constant such that $q^{\prime}=c q .^{5}$ More generally, $q, q^{\prime}$ are related to some smooth diffeomorphism.

As a converse remark, notice that if $\Sigma$ were not separable or that $\gamma_{q}=\left\{\gamma_{q}^{i} \mid i \in \mathbb{N}\right\}$ were not chosen to satisfy (2), $\gamma_{q}$ need not uniquely determine $[q] \in \mathscr{Q}$. Call $\mathscr{M}_{\infty}\left[\Gamma_{2}^{+}\right] \stackrel{\text { def }}{=} \bigcup_{q \in \Gamma_{2}^{+}} \mathscr{M}_{\infty}[q]$ the space of piecewise geodesic $\aleph_{0}$-loops. A suitable topology will be constructed on this space below.

Let $L_{\Sigma}\left[\Gamma_{2}^{+}\right]$denote the set of all affinely parametrized, piecewise geodesic loops in $\Sigma$ and let $L_{\Sigma}^{\infty}\left[\Gamma_{2}^{+}\right]$denote the countably infinite (set-theoretic) product of $L_{\Sigma}\left[\Gamma_{2}^{+}\right]$. Define an equivalence relation $R_{\infty} \subset L_{\Sigma}^{\infty}\left[\Gamma_{2}^{+}\right] \times L_{\Sigma}^{\infty}\left[\Gamma_{2}^{+}\right]$by $R_{\infty}=\left\{\left(\gamma, \gamma^{\prime}\right):[\gamma]=\right.$ $\left.\left[\gamma^{\prime}\right]\right\}$, where $[\eta] \stackrel{\text { def }}{=}\left\{\eta^{i} \in L_{\Sigma}\left[\Gamma_{2}^{+}\right]: \eta=\left(\eta^{i}\right)_{i=1}^{\infty}\right\}$ is just the set of components of the $\aleph_{0}$-loop $\eta$. Let $\pi_{\Sigma}: L_{\Sigma}^{\infty}\left[\Gamma_{2}^{+}\right] \rightarrow \mathscr{M}\left[\Gamma_{2}^{+}\right] \stackrel{\text { def }}{=} \mathscr{L}_{\Sigma}^{\infty}\left[\Gamma_{2}^{+}\right] / R_{\infty}$ be the natural map. If $\mathscr{M}_{n}\left[\Gamma_{2}^{+}\right]$denotes the set of (affinely parametrized) piecewise geodesic $n$-loops, then $\mathscr{M}\left[\Gamma_{2}^{+}\right] \equiv \bigcup_{n=1}^{\infty} \mathscr{M}_{n}\left[\Gamma_{2}^{+}\right]$. Now, let $M_{\infty} \subset \mathscr{L}_{\Sigma}^{\infty}\left[\Gamma_{2}^{+}\right]$be a subset satisfying

(a) for each $\gamma \stackrel{\text { def }}{=}\left(\gamma^{i}\right)_{i=1}^{\infty} \in M_{\infty}, \gamma^{i} \neq \gamma^{j} \quad \forall i \neq j$,

(b) $\pi_{\Sigma}\left(M_{\infty}\right)=\mathscr{M}_{\infty}\left[\Gamma_{2}^{+}\right] \subset \mathscr{M}\left[\Gamma_{2}^{+}\right]$.

It is clear from the definition of $M_{\infty}$ that there exists a family of subsets $M_{\sigma} \subset M_{\infty}$ satisfying

(i) $M_{\infty}=\bigcup_{\sigma} M_{\sigma}$,

(ii) $M_{\sigma} \cap M_{\sigma^{\prime}}=\emptyset \forall \sigma \neq \sigma^{\prime}$,

(iii) $\pi_{\Sigma} \mid M_{\sigma}: M_{\sigma} \rightarrow \mathscr{M}_{\infty}\left[\Gamma_{2}^{+}\right]$is a bijection.

Let $h_{\sigma} \stackrel{\text { def }}{=} \pi_{\Sigma} \mid M_{\sigma}$ and for each $\gamma \in \mathscr{M}_{\infty}\left[\Gamma_{2}^{+}\right]$, set $\gamma_{\sigma}=h_{\sigma}^{-1}(\gamma) \in M_{\sigma}{ }^{6}$ The subsets $M_{\sigma}$ can be endowed with a metric topology. A metric on $M_{\sigma}$ will now be constructed. Firstly, fix a finite atlas $\mathfrak{U}$ on $\Sigma$. Secondly, note that if $\Omega_{\Sigma}=\{\gamma$ : $I \rightarrow \Sigma \mid \gamma(0)=\gamma(1), \gamma$ continuous] denotes the loop space of $\Sigma$ and if $d_{q}$ is a (topological) metric on $\Sigma$ (induced by a Riemannian 3-metric $q$ ) compatible with its manifold topology, then $d_{\Omega}(\gamma, \eta) \stackrel{\text { def }}{=} \sup _{t \in I} d_{q}(\gamma(t), \eta(t))$ defines a metric on $\Omega_{\Sigma}$ compatible with its compact-open topology. ${ }^{?}$

Now, given a pair of $\aleph_{0}$-loops $\gamma, \eta \in M_{\sigma}$, let

$$
d_{\Omega}^{\prime}\left(\gamma^{i}, \eta^{i}\right) \stackrel{\text { def }}{=} \operatorname{ess} \sup \left\{\left\|D^{k} \gamma^{i}(t)-D^{k} \eta^{i}(t)\right\|: t \in I, k \geqq 1\right\},
$$

where sup runs over all relevant (finite) charts $(U, \varphi) \in \mathfrak{U}$, ess denoting that the expression $\left\|D^{k} \gamma^{i}(t)-D^{k} \eta^{i}(t)\right\|$ is defined on $I$ a.e. - that is, it is not defined only on a finite (possibly zero) set of points in $I$ wherein $\gamma^{i}$ and $\eta^{i}$ are not differentiable,

\footnotetext{
${ }^{5}$ Note trivially that as $q, q^{\prime}$ are positive-definite, $c<0$ is not an admissible solution.

${ }^{6}$ The subscript $\sigma$ on $\gamma_{\sigma}$ will be omitted if no confusion should arise from the context.

${ }^{7}$ Observe trivially that the $d_{\Omega}$-topology does not depend on the choice of the (admissible) 3-metric $q$ since all (topological) metrics on $\Sigma$ induced by (admissible) Riemannian 3-metrics $q$ are equivalent.
} 
and $D^{k} \gamma^{i}(t)$ denotes the $k$ th differential of $\gamma^{i}$ at $t$ in abused notations. Finally, set $d_{\sigma}(\gamma, \eta) \stackrel{\text { def }}{=} \sup _{i} d_{\Omega}\left(\gamma^{i}, \eta^{i}\right)+\sup _{i} d_{\Omega}^{\prime}\left(\gamma^{i}, \eta^{i}\right)$. It is routine to verify that $d_{\sigma}$ is indeed a metric on $M_{\sigma}$.

REMARK 2.2. It can be shown that the $d_{\sigma}$-topology is compatible with the topology on $M_{\sigma}$ generated by the subbasic sets $N_{\varepsilon}\left(\gamma ;\left(U_{\alpha(i)}, \varphi_{\alpha(i)}\right)_{i=1}^{\infty}, K\right)$ to be defined below, where $K \subset I$ is compact, $\gamma^{i}(K) \subset U_{\alpha(i)}$, and $\left(U_{\alpha(i)}, \varphi_{\alpha(i)}\right) \in \overline{\mathfrak{U}}$ for each $i$, with $\overline{\mathfrak{U}}$ being the maximal atlas of $\Sigma$. Firstly, set $\alpha \stackrel{\text { def }}{=}\{\alpha(i) \mid 1 \leqq i \leqq \infty\}$ and denote $\left(U_{\alpha(i)}, \varphi_{\alpha(i)}\right)_{i}$ by $(U, \varphi)_{\alpha}$ for notational convenience. Next, let

$$
d_{\sigma \alpha K}^{\prime}\left(\gamma^{i}, \eta^{i}\right) \stackrel{\text { def }}{=} \operatorname{ess} \sup \left\{\left\|D^{k} \varphi_{\alpha(i)} \circ \gamma^{i}(t)-D^{k} \varphi_{\alpha(i)} \circ \eta^{i}(\ddot{t})\right\|: t \in K, k \geqq 1\right\}
$$

whenever $\gamma^{i}(K), \eta^{i}(K) \subset U_{\alpha(i)} \forall i$. Then, for a fixed $\gamma \in M_{\sigma}$ such that $\gamma^{i}(K) \subset$ $U_{\alpha(i)} \forall i$, let $N_{\varepsilon}\left(\gamma ;(U, \varphi)_{\alpha}, K\right) \stackrel{\text { def }}{=}\left\{\eta \in M_{\sigma} \mid \bar{d}_{\sigma \alpha K}(\gamma, \eta)<\varepsilon, \eta^{i}(K) \subset U_{\alpha(i)} \forall i\right\}$, where

$$
\bar{d}_{\sigma \alpha K}(\gamma, \eta) \stackrel{\text { def }}{=} \sup _{i} d_{\Omega}\left(\gamma^{i}, \eta^{i}\right)+\sup _{i} d_{\sigma \alpha K}^{\prime}\left(\gamma^{i}, \eta^{i}\right) .
$$

In particular, the $d_{\sigma}$-topology does not depend on the particular choice of (admissible) finite atlas $\mathfrak{U}$ of $\Sigma$. Hence, in this sense, the $d_{\sigma}$-topology is well-defined.

It is easy to see from the construction that $h_{\sigma \sigma^{\prime}}: M_{\sigma} \rightarrow M_{\sigma^{\prime}}$ given by $\gamma_{\sigma} \mapsto \gamma_{\sigma^{\prime}}$, where $h_{\sigma}\left(\gamma_{\sigma}\right)=\gamma=h_{\sigma^{\prime}}\left(\gamma_{\sigma^{\prime}}\right)$, defines a homeomorphism. The existence of $h_{\sigma \sigma^{\prime}}$ follows immediately from properties (a) and (iii) above. Hence, it is possible to endow $\mathscr{M}_{\infty}\left[\Gamma_{2}^{+}\right]$with a topology so that each $h_{\sigma}: M_{\sigma} \rightarrow \mathscr{M}_{\infty}\left[\Gamma_{2}^{+}\right]$defines a homeomorphism. In this paper, $\mathscr{M}_{\infty}\left[\Gamma_{2}^{+}\right]$will be equipped with this topology. As an aside, if $M_{\infty}$ were given the sum topology, $M_{\infty} \stackrel{\text { def }}{=} \bigoplus_{\sigma} M_{\sigma}$, then $h: M_{\infty} \rightarrow \mathscr{M}_{\infty}\left[\Gamma_{2}^{+}\right]$ defined by $h \mid M_{\sigma}=h_{\sigma}$ is a continuous open surjection.

\section{Knots and 3-geometries}

First of all, recall that a smooth ambient isotopy is a smooth deformation of one loop into another such that the surrounding manifold is smoothly transformed. More precisely, it is a smooth map $F: \Sigma \times I \rightarrow \Sigma \times I$ given by $(x, t) \mapsto\left(F_{t}(x), t\right)$ such that $F_{0}=\operatorname{id}_{\Sigma}$ and $F_{t} \in \operatorname{Diff}(\Sigma) \forall t \in I$. Let $\mathscr{G}_{a}^{+} \subset C^{\infty}(\Sigma \times I, \Sigma \times I)$ be the set of (smooth) orientation-preserving, ambient isotopies on $\Sigma$.

If $\gamma, \eta \in \mathscr{L}_{\Sigma}$ are any pair of loops and $\gamma$ is ambiently isotopic to $\eta$ under some $F \in \mathscr{G}_{a}^{+}$, denote this by $F: \gamma \simeq \eta$. Now, given any pair of $\aleph_{0}$-loops $\gamma, \eta \in \mathscr{M}_{\infty}\left[\Gamma_{2}^{+}\right]$, define an equivalence relation $R$ generated by $\simeq$ on $\mathscr{M}_{\infty}\left[\Gamma_{2}^{+}\right]$as follows:

$$
\gamma \simeq \eta \quad \Longleftrightarrow \quad \exists F \in \mathscr{G}_{a}^{+} \text {such that } F \cdot \gamma=\eta,
$$


where $F \cdot \gamma \stackrel{\text { def }}{=}\left\{F_{1} \circ \gamma^{1}, F_{1} \circ \gamma^{2}, \ldots\right\}$ and $F: \gamma^{i} \simeq \eta^{i} \forall i$. Then the space $\mathscr{K}\left[\Gamma_{2}^{+}\right]$ of equivalence classes of $\kappa_{0}$-loops in $\mathscr{M}_{\infty}\left[\Gamma_{2}^{+}\right]$is defined to be the quotient space $\mathscr{M}_{\infty}\left[\Gamma_{2}^{+}\right] / R$. Henceforth, for simplicity, call an element $[\gamma] \stackrel{\text { def }}{=}\left\{\eta \in \mathscr{M}_{\infty}\left[\Gamma_{2}^{+}\right]\right.$: $\eta \simeq \gamma\}$ of the quotient space $\mathscr{K}\left[\Gamma_{2}^{+}\right]$a (piecewise geodesic) $\kappa_{0}$-knot and let $\kappa_{\infty}$ : $\mathscr{M}_{\infty}\left[\Gamma_{2}^{+}\right] \rightarrow \mathscr{K}\left[\Gamma_{2}^{+}\right]$denote the natural map. In the interest of simplicity, call $\gamma \in \mathscr{M}_{\infty}\left[\Gamma_{2}^{+}\right]$a piecewise $\left(\aleph_{0}, q\right)$-geodesic loop whenever the 3-metric $q$ is required to be specified.

LEMMA 3.1. Let $\gamma, \tilde{\gamma} \in \mathscr{M}_{\infty}\left[\Gamma_{2}^{+}\right]$be piecewise $\left(\aleph_{0}, q\right)$-and $\left(\aleph_{0}, \tilde{q}\right)$-geodesic loops respectively. If $\gamma \simeq \tilde{\gamma}$, then $\exists f \in \operatorname{Diff}^{+}(\Sigma)$ such that $q=f^{*} \tilde{q}$.

Proof. Let $F \in \mathscr{G}_{a}^{+}$be an ambient isotopy of $\gamma$ and $\tilde{\gamma}: F \cdot \gamma=\tilde{\gamma}$. Then, evidently, $\tilde{\gamma}$ is a piecewise $\left(\aleph_{0},\left(F_{1}^{-1}\right)^{*} q\right)$-geodesic. However, $\tilde{\gamma}$ is also a piecewise $\left(\aleph_{0}, \tilde{q}\right)$-geodesic; hence, by $(2), \exists f \in \operatorname{Diff}^{+}(\Sigma)$ such that $q=f^{*} \tilde{q}$, as required.

The main results of this paper will now be stated. In fact, the correspondence between loops and geometries can be easily sought simply by noting that each element in $\mathscr{M}_{\infty}\left[\Gamma_{2}^{+}\right]$corresponds to a unique 3 -geometry $[q]$ of $\Sigma$ by construction.

THEOREM 3.2. There exists a continuous, open surjection $\hat{\chi}: \mathscr{M}_{\infty}\left[\Gamma_{2}^{+}\right] \rightarrow \mathscr{Q}$ given by $\gamma_{q} \mapsto[q]$, where $\gamma_{q}$ is a (piecewise) $\left(\aleph_{0}, \tilde{q}\right)$-geodesic loop and $q \in[q]$.

PROOF. The details can be found in [7, Theorem 4.1].

COROLLARY 3.3. The map $\hat{\chi}$ induces a continuous, open surjection $\chi: \mathscr{K}\left[\Gamma_{2}^{+}\right] \rightarrow$ $\mathscr{Q}$ given by $\left[\gamma_{q}\right] \mapsto \hat{\chi}\left(\gamma_{q}\right)$, where $\gamma_{q} \in \kappa_{\infty}^{-1}\left(\left[\gamma_{q}\right]\right)$ is any fixed representative.

Proof. The map $\chi$ is well-defined by Lemma 3.1. The result now follows immediately from Theorem 3.2, the openness of the projection map $\kappa_{\infty}$ and from the commutativity of the following diagram:

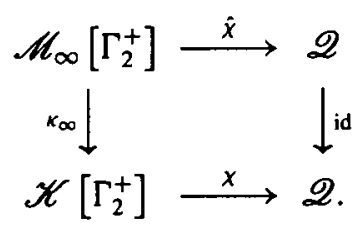

\section{Discussion}

It is easy to observe from Theorem 3.2 that at the classical level, each $\aleph_{0}$-loop $\gamma \in \mathscr{M}_{\infty}\left[\Gamma_{2}^{+}\right]$contains enough information to restrict the 3-manifold $\Sigma$ together with 
its 3-geometry $[q]$. To see this, it is sufficient to note firstly that $\chi$ maps $\gamma$ to a unique 3-geometry $[q]$. Then, by choosing any representative of $[q]$ and defining the closure of $\bigcup\left\{\gamma^{i}(I) \mid \gamma^{i} \in \gamma\right\}$ with respect to the metric induced by $q$ yields the Riemannian manifold $(\Sigma, q)$.

This in turn suggests that $\aleph_{0}$-loops are suitable candidates for the description of gravitational states. Heuristically, we may interpret a knot state $|[\gamma]\rangle,[\gamma] \in \mathscr{K}\left[\Gamma_{2}^{+}\right]$, as a state associated with a 3-manifold together with its Riemannian 3-geometry $(\Sigma, \chi([\gamma]))$. That is, each knot state $|[\gamma]\rangle$ corresponds to the global degrees of freedom of gravity. Secondly, functionals on $\mathscr{L}_{\Sigma}$ which describe gravitational states are constant on the $\mathscr{G}_{a}^{+}$-orbits of $\mathscr{L}_{\Sigma}-\psi[\gamma]=\psi\left[\gamma^{\prime}\right] \forall \gamma, \gamma^{\prime} \in[\gamma]$, where $\psi: \mathscr{L}_{\Sigma} \rightarrow$ $\mathbb{C}$ is a loop functional — due to the diffeomorphism constraint of general relativity (in the loop representation) [6, p. 132]. Surprisingly, this condition follows immediately from Corollary 3.3. For let $C(\mathscr{Q}, \mathbb{C})$ be the set of continuous functionals on $\mathscr{Q}$ and $C\left(\mathscr{K}\left[\Gamma_{2}^{+}\right], \mathbb{C}\right)$ that of $\mathscr{K}\left[\Gamma_{2}^{+}\right]$. Then, $\forall \tilde{\Psi} \in C(\mathscr{Q}, \mathbb{C}), \tilde{\Psi} \circ \chi \in C\left(\mathscr{K}\left[\Gamma_{2}^{+}\right], \mathbb{C}\right) ;$ that is, $\chi^{*}(C(\mathscr{Q}, \mathbb{C})) \subset C\left(\mathscr{K}\left[\Gamma_{2}^{+}\right], \mathbb{C}\right)$, and the assertion thus follows.

This paper will conclude by outlining a prime motivation for studying the relationship between knots and geometries. It is possible to show, by relaxing the bijective condition of (2) imposed on $\gamma$ - that is, $\gamma^{i} \mapsto \gamma^{i}(0)$ is a bijection - to a surjective one, and by imposing additional conditions on the $\aleph_{0}$-loops, that the resulting $\aleph_{0}$-loop space $\mathscr{M}_{\infty}$ admits a smooth manifold structure modelled on a locally convex topological vector space. This has the implication that $\mathscr{M}_{\infty}$ can be regarded as a configuration space for gravity in the sense of geometric quantization. Thus, in this sense, $\mathscr{M}_{\infty}$ has the interpretation of being the 'dynamical' space where 3-geometries evolve. This is of course rather speculative, and work in this area is currently in progress.

\section{Acknowledgement}

The first author thanks L. Tassie and S. Scott for some helpful conversations.

\section{References}

[1] A. Ashtekar, "New Hamiltonian formulation of general relativity", Phys. Rev. D36 (1987) 15871602.

[2] M. P. Blencowe, "The Hamiltonian constraint in quantum gravity", Nucl. Phys. B341 (1990) 213251.

[3] B. Brügmann and J. Pullin, "On the constraints of quantum gravity in the loop representation", Nucl. Phys. B390 (1993) 399-438.

[4] T. Jacobson and L. Smolin, "Nonperturbative quantum geometries", Nucl. Phys. B299 (1988) 295345. 
[5] C. Rovelli, "Ashtekar's formulation of general relativity and loop-space non-perturbative quantum gravity: a report", Class. Quantum Grav. 8 (1991) 1613-1675.

[6] C. Rovelli and L. Smolin, "Loop representation of quantum general relativity", Nucl. Phys. B331 (1990) 80-152.

[7] T.-C. Toh and M. R. Anderson, "Knots and classical 3-geometries", J. Math. Phys. 36 (1995) 596-604. 